\title{
The Coverage Analysis for Low Earth Orbiting Satellites at Low Elevation
}

\author{
Shkelzen Cakaj ${ }^{1}$, Bexhet Kamo ${ }^{1}$, Algenti Lala ${ }^{1}$, Alban Rakipi ${ }^{1}$ \\ ${ }^{1}$ Faculty of Information Technology \\ Polytechnic University of Tirana \\ Tirana, Albania
}

\begin{abstract}
Low Earth Orbit (LEO) satellites are used for public networking and for scientific purposes. Communication via satellite begins when the satellite is positioned in its orbital position. Ground stations can communicate with LEO satellites only when the satellite is in their visibility region. The duration of the visibility and the communication vary for each LEO satellite pass over the station, since LEO satellites move too fast over the Earth. The satellite coverage area is defined as a region of the Earth where the satellite is seen at a minimum predefined elevation angle. The satellite's coverage area on the Earth depends on orbital parameters. The communication under low elevation angles can be hindered by natural barriers. For safe communication and for savings within a link budget, the coverage under too low elevation is not always provided. LEO satellites organized in constellations act as a convenient network solution for real time global coverage. Global coverage model is in fact the complementary networking process of individual satellite's coverage. Satellite coverage strongly depends on elevation angle. To conclude about the coverage variation for low orbiting satellites at low elevation up to $10^{\circ}$, the simulation for attitudes from $600 \mathrm{~km}$ to $1200 \mathrm{~km}$ is presented through this paper.
\end{abstract}

Keywords—LEO; satellite; coverage

\section{INTRODUCTION}

Generally, satellite' circular orbits are categorized as Geosynchronous Earth Orbits (GEO), Medium Earth Orbits (MEO) and Low Earth Orbits (LEO). The main difference among them is in the attitude above the Earth surface [1]-[3].

The satellites traversing in orbits of attitudes up to around $1400 \mathrm{~km}$ (limited by Van Allen belt [4]) are considered as LEO satellites. LEO satellites are moving at around $7.5 \mathrm{~km} / \mathrm{s}$ velocity relative to a fixed point on the Earth (ground station) [5]. The characteristics of LEOs are: the shortest distance from the Earth compared with other orbits and consequently less time delay. These characteristics make them very attractive even for scientific applications or communications networking [5], [6].

The single satellite coverage area is defined as a region of the Earth where the satellite is seen at a minimum predefined elevation angle. For multi satellite coverage or global coverage the management policy for satellite coordination must be applied. For global coverage, handover and management policies become more critical under too low elevation because of natural barriers. Handover policies and management are well analyzed under [7] and [8].

Analysis of random coverage time in mobile LEO satellite communications is also well treated by [9].

This paper discusses the single LEO satellite coverage aspects as an overture to the global coverage. Some characteristics of LEO satellites are given followed by coverage geometry. Finally the results of coverage simulation under different attitudes from $600 \mathrm{~km}$ to $1200 \mathrm{~km}$ at low elevation are presented.

\section{LEO SATELLITES AND COVERAGE}

Microsatellites in Low Earth Orbits (LEO) have been in use for the past two decades, mainly dedicated for scientific purposes. LEO satellites have very wide scientific applications, from remote sensing of oceans, through analyses on Earth's climate changes, Earth's imagery with high resolution or astronomical purposes. These satellites provide opportunity for investigations for which alternative techniques are either difficult or impossible to be applied. Thus, it may be expected that such scientific missions will be further developed in the near future especially in fields where similar experiments by purely Earth-based means are impracticable. Ground stations have to be established in order to communicate with such satellites. Ground stations can communicate with LEO (Low Earth Orbiting) satellites only when the satellite is in their visibility region.

Satellites in these orbits have an orbital period of around (90-110) minutes. For satellites this is a short flyover period, which means that the antenna at the ground station must follow the satellite very fast with high pointing accuracy. The contact communication time between the satellite and the ground station takes (5-15) minutes 6-8 times during the day [6]. The Hubble Space Telescope, for example, operates at an altitude of about $610 \mathrm{~km}$ with an orbital period of 97 minutes [6]. Every satellite (especially, microsatellite when is dedicated for scientific purposes) carries special instruments that enable it to perform its mission [5] (for example, a satellite that studies the universe has a telescope, a satellite that helps forecast the weather carries cameras to track the movement of clouds).

On other hand from the communication perspective the goal of the future communication systems is to provide high quality broadband services with global coverage [10]. The satellite constellation is a convenient network solution for real time global coverage. The constellation is a system of low Earth orbit (LEO) identical satellites, launched in several orbital planes with the orbits having the same altitude. The 
satellites move in a synchronized manner in trajectories relative to Earth. The application of low Earth orbit satellites organized in a constellation is an alternative to wireless telephone networks. Satellites in low orbits arranged in a constellation, work together by relaying information to each other and to the users on the ground. If satellites within a constellation are equipped with advanced on-board processing, they can communicate directly with each other by line of sight using inter-satellite links (ISL) [5].

To provide a global coverage to a diverse user population a number of LEO satellite networks have been proposed and implemented. The LEO satellite networks can support both the areas with terrestrial wireline and wireless networks that lack any network infrastructure [7]. Nowadays Several LEO constellations (Globalstar, Iridium, Ellipso) are active and operational [7], [8]. For complete coverage of the Earth's surface some overlapping between the adjacent satellites is necessary, to keep the continuity of real time services [9]. The global coverage can be considered as an interoperable complementary networking process of multiple satellites organized in constellation, each of them contributing with its individual coverage. This is achieved because LEO satellites move with respect to a fixed observer on Earth surface, and along with satellite movement also the coverage area changes its position continually creating a coverage belt as in Fig.1. Satellites under the same attitude under different inclination make different belts, enabling global coverage. Individual satellite coverage for few LEO satellites is presented in Fig. 2.

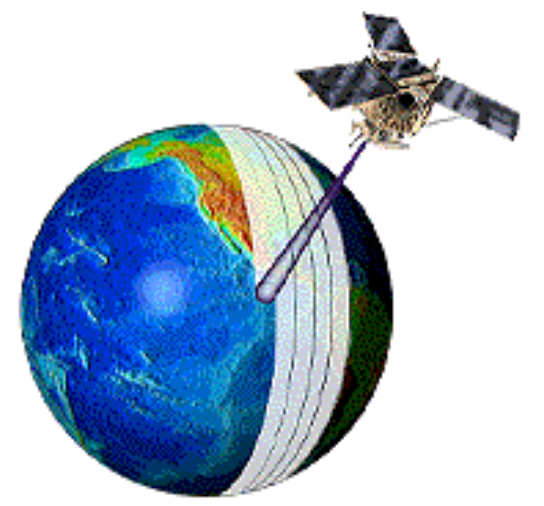

Fig. 1. Few LEO coverage area (Source: NOAA, 2009)

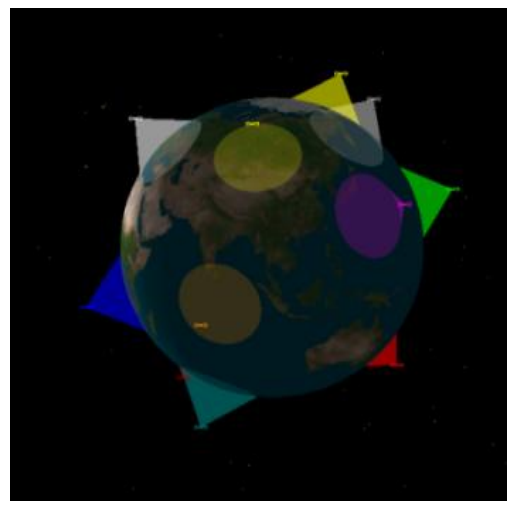

Fig. 2. Few LEOs coverage area (Source: NOAA, 2009)
When designing a satellite network some decisions such as the selection of the orbit parameters, coverage model, the network connectivity and routing model must be made. LEO satellite networks as Iridium, Teledesic, Globalstar, have architecture differences affecting the capabilities and services. The main problem for the global coverage is the handover process from one coverage area to another one. This is called satellite hand over process [7].

Different deterministic models for coverage time evaluation of low Earth orbiting satellite are developed. Models involve statistical coverage time assessments [8]. The analyses are particularly useful for probabilistic investigation of intersatellite handovers in LEO satellite networks [8]. The probability of service interruption and hand over mechanism becomes important for the overall system performance [9].

Achievements in antenna technology leaded to multibeam LEO systems where the footprint or coverage area is divided in many cells (multibeam arrays) in order to enhance frequency reuse policies. Applying space diversity policy is achieved frequency reuse inside a footprint. Handover from one cell to another is defined as cell handover. Particularly the interference problems have carefully to be treated [11] - [13].

\section{LEO COVERAGE GEOMETRY}

The position of the satellite within its orbit considered from the ground station point of view is defined by Azimuth $(A z)$ and Elevation $\left(\varepsilon_{0}\right)$ angles. The azimuth is the angle of the direction of the satellite, measured in the horizon plane from geographical north in clockwise direction. The range of azimuth is $0^{\circ}$ to $360^{\circ}$. The elevation is the angle between a satellite and the observer's (ground station's) horizon plane. The range of elevation is $0^{\circ}$ to $90^{\circ}$.

The coverage area of a single satellite is a circular area (Fig. 2) on the Earth surface in which the satellite can be seen under an elevation angle equal or greater than the minimum elevation angle determined by the link budget requirements of the system. The largest coverage area is achieved under elevation of $0^{\circ}$, but in order to avoid obstacles caused by natural barriers at too low elevation, usually for the link budget calculations it is determined the minimal elevation angle which ranges on (2-10) ${ }^{\mathbf{0}}$. For simulation purposes of coverage it is considered the elevations up to $10^{\circ}$.

The satellite's coverage area on the Earth depends on orbital parameters. Ground stations (GS) can communicate with LEO (Low Earth Orbiting) satellites only when the ground station is under coverage area (satellite footprint) as presented in Fig. 3.

The duration of the visibility and consequently the communication duration vary for each LEO satellite pass over the ground station, since LEO satellites move too fast over the Earth. Along with satellite, the footprint moves also, leaving the GS out of the footprint and consequently loosing the communication with the GS, as presented in Fig. 4. 


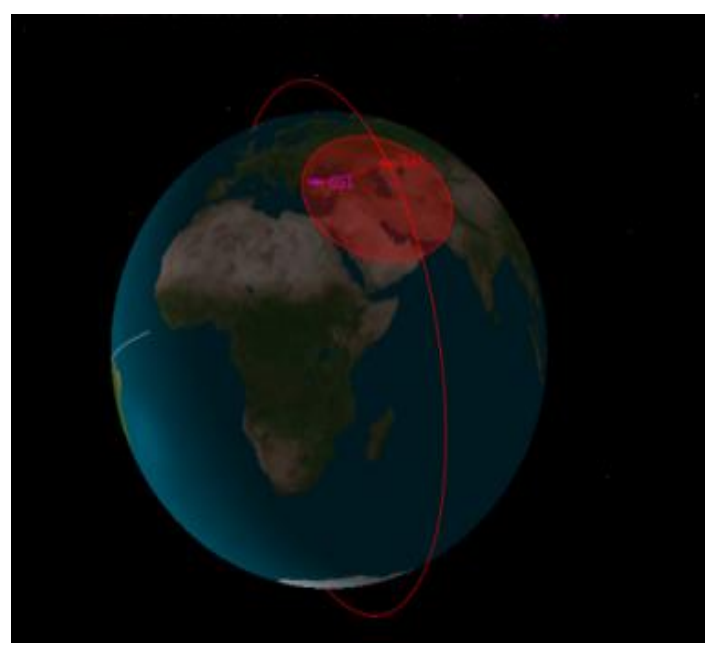

Fig. 3. The ground station (GS) under the LEO coverage area.

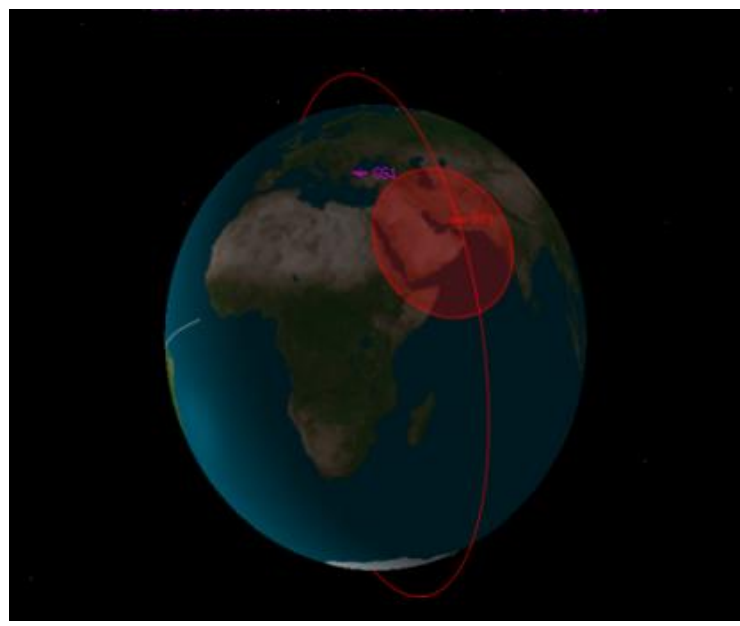

Fig. 4. The ground station (GS) out off the LEO coverage area.

The basic geometry between a satellite and ground station is depicted in Fig. 5. The points indicate the satellite (SAT), ground station (P), and then the third is the Earth's center. The line passing at point $\mathrm{P}$ represents horizon plane. The subsatellite point is indicated by T. Two sides of this triangle are usually known (the distance from the ground station to the Earth's center, $R_{E}=6378 \times 10^{3} \mathrm{~m}$ and the distance from the satellite to Earth's center-orbital radius). There are four variables in this triangle: $\varepsilon_{0}$ - is elevation angle, $\alpha_{0}$ - is nadir angle, $\beta_{0}$ - is central angle and $d$ is slant range. As soon as two quantities are known, the others can be found with the following equations [14]:

$$
\begin{gathered}
\varepsilon_{0}+\alpha_{0}+\beta_{0}=90 \\
d \cos \varepsilon_{0}=r \sin \beta_{0} \\
d \sin \alpha_{0}=R_{e} \sin \beta_{0}
\end{gathered}
$$

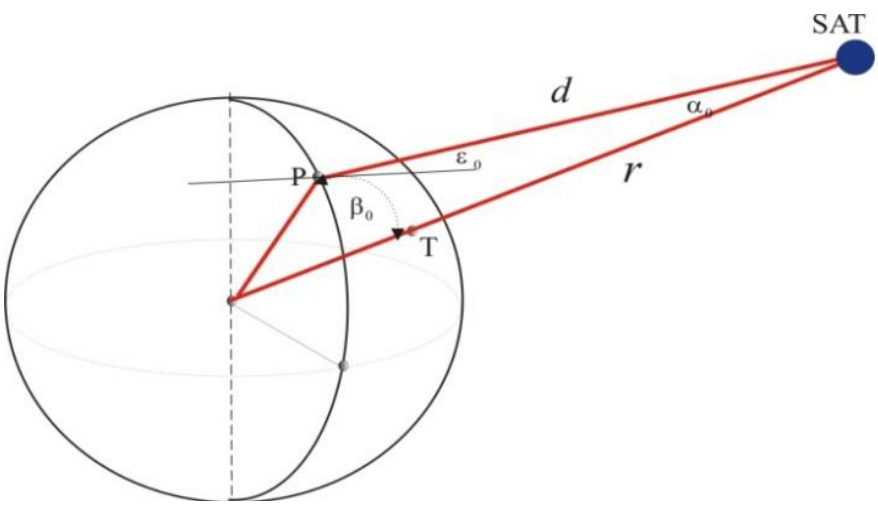

Fig. 5. Ground station geometry

The most needed parameter is the slant range $d$ (distance from the ground station to the satellite). This parameter will be used during the link budget calculation, and it is expressed through elevation angle $\varepsilon_{0}$. Applying cosines law for triangle at Fig. 5 yields out:

$$
r^{2}=R_{e}^{2}+d^{2}-2 R_{e} d \cos \left(90+\varepsilon_{0}\right)
$$

Solving (4) by $d$, substituting, $\quad r=H+R_{e}$ at (5) and applying (1), (2), (3) finally we will get the slant range as function of elevation angle $\varepsilon_{0}$ [14].

$$
d\left(\varepsilon_{0}\right)=R_{e}\left[\sqrt{\left(\frac{H+R_{e}}{R_{e}}\right)^{2}-\cos ^{2} \varepsilon_{0}}-\sin \varepsilon_{0}\right]
$$

$H$ is the satellite attitude above the Earth's surface. Transforming Fig. 5 from the coverage point of view it looks like in Fig. 6.

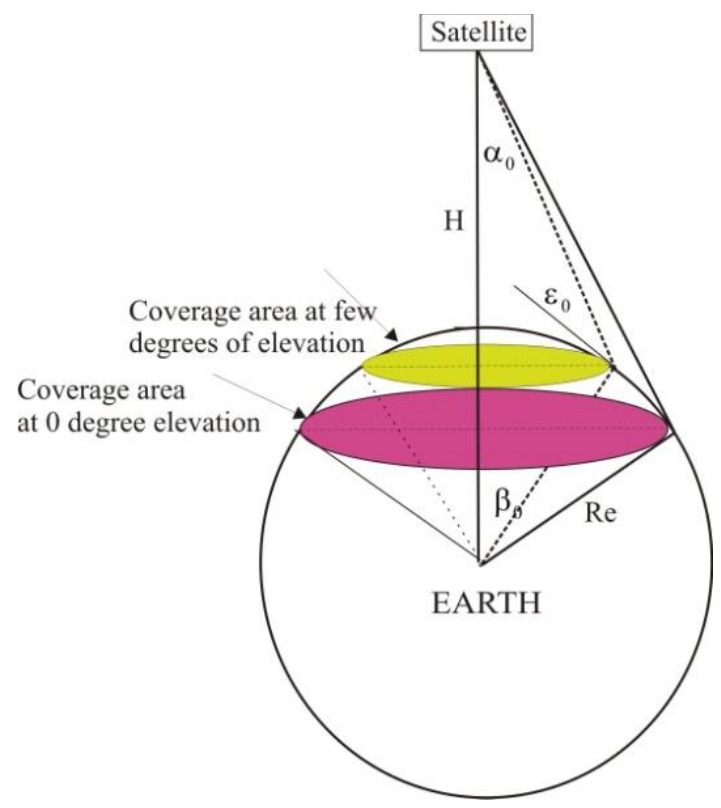

Fig. 6. Coverage geometry 
In Fig. 6, there are two triangles. The larger one represents the case of full coverage under elevation of $0^{\circ}$. Generally, from the smaller triangle applying sinus theorem yields out:

$$
\begin{aligned}
& \frac{\sin \alpha_{0}}{R_{e}}=\frac{\sin \left(90+\varepsilon_{0}\right)}{R_{e}+H} \\
& \sin \alpha_{0}=\frac{R_{e}}{R_{e}+H} \cos \varepsilon_{0}
\end{aligned}
$$

The maximal coverage is achieved for $\varepsilon_{0}=0$, thus for known attitude $H$, easy is calculated the coverage angle for maximal coverage, as:

$$
\left(\sin \alpha_{0}\right)_{M A X, H}=\frac{R_{e}}{R_{e}+H}
$$

Similarly, for different elevations $\left({ }^{\varepsilon_{0}}\right)$ can be calculated ${ }^{\alpha_{0}}$ and then based on (1) also $\beta_{0}$.

The surface of the coverage area depends on $\beta_{0}$ angle and it is [15]:

$$
S_{\text {Coverage }}=2 \pi R_{e}^{2}\left(1-\cos \beta_{0}\right)
$$

Usually, the satellite coverage area or the satellite's footprint is expressed (in percentage) as a fraction of the Earth's area.

$$
\text { Coverage }(\%)=\frac{S_{\text {Coverage }}}{S_{\text {Earth }}}=\frac{2 \pi R_{e}^{2}\left(1-\cos \beta_{0}\right)}{4 \pi R_{e}^{2}}
$$

$$
\text { Coverage }(\%)=\frac{1}{2}\left(1-\cos \beta_{0}\right)
$$

\section{LEO COVERAGE SimUlation}

Based on (7), (8) and (11) it is obvious that the satellite coverage strongly depends on elevation angle. To conclude about the coverage variation for low orbiting satellites at low elevation, the simulation for attitudes from $600 \mathrm{~km}$ up to $1200 \mathrm{~km}$ is further discussed.

For a given satellite attitude $H$ and a given elevation angle $\varepsilon_{0}$ firstly should be calculated $\alpha_{0}, \beta_{0} \quad$ and finally the coverage based on (12). For attitudes of $H=600 \mathrm{~km}, 800 \mathrm{~km}$, $1000 \mathrm{~km}$ and $1200 \mathrm{~km}$ which are typical low orbit attitudes it is simulated and calculated the coverage area for elevation of $(0-$ $10)^{\circ}$ by steps of $2^{\circ}$, and results are presented in Table I and Fig. 7.

Table I and Fig. 7 confirm the decrease of coverage area as elevation angle increases for the already defined attitude $H$, and the increase of the coverage area as attitude $H$ increases keeping the fixed elevation.
TABLE I. COVERAGE ARE AS A Fraction OF EARTH AREA.

\begin{tabular}{|c|c|c|c|c|}
\hline $\begin{array}{c}\text { Orbital } \\
\text { Attitude } \\
{[\mathbf{k m}]}\end{array}$ & $\begin{array}{c}\mathbf{H} \\
\mathbf{6 0 0} \\
{[\mathbf{k m}]}\end{array}$ & $\begin{array}{c}\mathbf{H} \\
\mathbf{8 0 0} \\
{[\mathbf{k m}]}\end{array}$ & $\begin{array}{c}\mathbf{H} \\
\mathbf{1 0 0 0} \\
{[\mathbf{k m}]}\end{array}$ & $\begin{array}{c}\mathbf{H} \\
\mathbf{1 2 0 0} \\
{[\mathbf{k m}]}\end{array}$ \\
\hline $\begin{array}{c}\text { Elevation } \\
\left(\boldsymbol{E}_{0}\right)\end{array}$ & $\begin{array}{c}\text { Coverage } \\
{[\%]}\end{array}$ & $\begin{array}{c}\text { Coverage } \\
{[\%]}\end{array}$ & $\begin{array}{c}\text { Coverage } \\
{[\%]}\end{array}$ & $\begin{array}{c}\text { Coverage } \\
{[\%]}\end{array}$ \\
\hline $0^{\circ}$ & 4.30 & 5.60 & 6.80 & 7.95 \\
\hline $2^{\circ}$ & 3.63 & 4.84 & 5.95 & 7.08 \\
\hline $4^{\circ}$ & 3.05 & 4.16 & 5.21 & 6.22 \\
\hline $6^{\circ}$ & 2.53 & 3.49 & 4.54 & 5.48 \\
\hline $8^{\circ}$ & 2.08 & 3.01 & 3.91 & 4.75 \\
\hline $10^{\circ}$ & 1.69 & 2.54 & 3.38 & 4.20 \\
\hline
\end{tabular}

The LEO coverage as a fraction of the Earth's surface

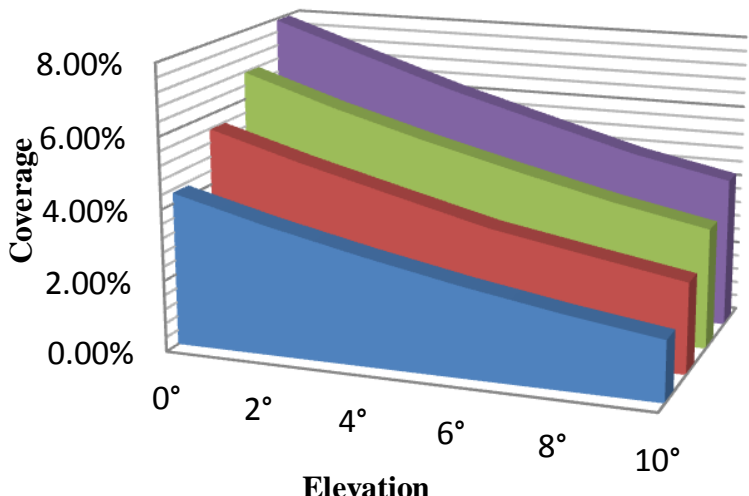

$600 \mathrm{Km}-800 \mathrm{Km}-1000 \mathrm{Km}-1200 \mathrm{Km}$

Fig. 7. Coverage area variation for different attitudes at low elevation

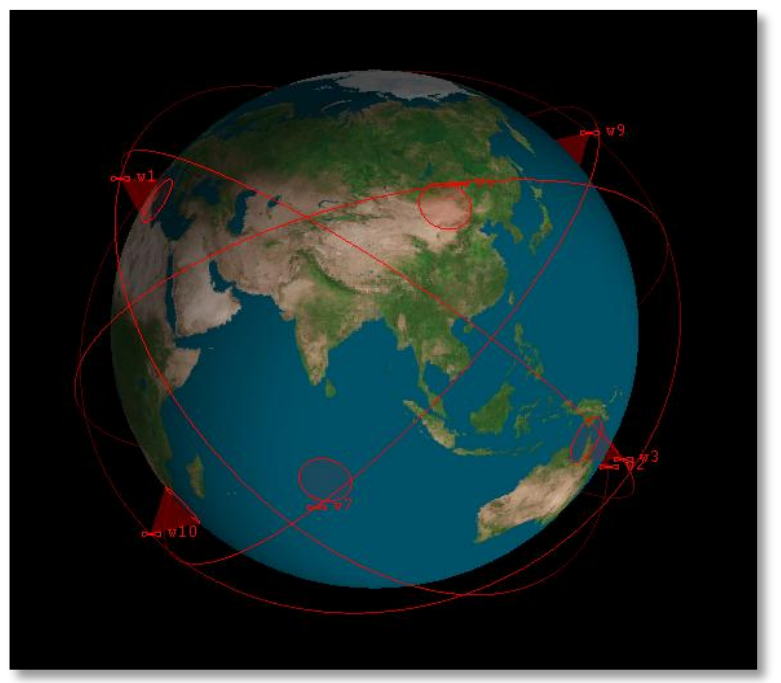

Fig. 8. LEO coverage area 
Finally, in Fig. 8, applying satellite orbit analysis software is presented the case of simulated coverage area for synchronized orbits at an attitude of $600 \mathrm{~km}$ for different inclination (few orbits) at elevation of $10^{\circ}$, as the smallest coverage area stemmed from the simulation considered in this paper. Small circles in Fig. 8 represent LEO coverage area on the Earth's surface.

For the real time services the main problem due to global coverage remains the handover process from one coverage area to another one. For real time services it is too important the reliable communication what can be disturbed under to low elevation because of natural barriers. Our future work will be oriented on correlation of single coverage area with GIS (Geographic Information System) in order to have the exact information under which elevation the safe communication could be provided. The simulation tools will be essential part of foreseen future work.

\section{CONCLUSION}

The satellite's coverage area on the Earth depends on orbital parameters and usually is expressed (in percentage) as a fraction of the Earth's area. LEO satellites organized in constellations act as a convenient solution for real time global coverage. Satellite coverage strongly depends on elevation angle. The largest coverage area is achieved under elevation of $0^{\circ}$, but in order to avoid obstacles caused by natural barriers at too low elevation, usually for the link budget calculations it is determined the minimal elevation angle which ranges on (2$10)^{\circ}$.

Through simulation for typical LEO attitudes on range of $(600-1200) \mathrm{km}$ at low elevation of $(0-10)^{\circ}$, it is confirmed that the fraction of Earth covered by satellites at appropriate attitudes is from $1.69 \%$ to $7.95 \%$.

\section{REFERENCES}

[1] M. Richharia, (1999), "Satellite communication systems", McGraw Hill, New York, 1999.

[2] D. Roddy, "Satellite communications", McGraw Hill, New York, 2006.

[3] G. Maral and M. Bousquet, "Satellite communication systems", John Willey \& Sons, Ltd, Chichester, England, 2002.

[4] www.answers.com/topic/van-allen-radiation -belt

[5] R. E. Zee, et al, "The MOST Microsatellite: A low cost enabling technology for future space science and technology missions", Canadian Aeronautics and Space Journal, 48(1), Canada, pg. 1-11, 2002.
[6] J.E. Oberright, "Satellite artificial", World Book Online Reference Center, World Book, Inc, 2004.

[7] I. F. Alyildiz, H. Uzunalioglu, M. D. Bender,Turkiye, " Handover management in Low Earth Orbit (LEO) satellite networks" Mobile Networks and Applications 4 (1999) 301 -310.

[8] P. Papapetrou, S. Karapantazis, F.N. Pavlidou, " Handover Policies in LEO Systems with Satellite Diversity", International Conference on Advanced Satellite Mobile Systems (ASMS 2003), 10-11 July, 2003, Frascati, Italy.

[9] Y. Seyedi, S. M. Safavi, "On the Analysis of Random Coverage Time in Mobile LEO Satellite Communications", Communications Letters, IEEE, Volume 16, Issue 5, MAY 2012.

[10] A. Botta, A. Pescape, "New generation satellite broadband Internet service: should ADSL and 3G worry", TMA 2013, co-lacted with IEEE INFOCOM 2013, April 2013, Turin, Italy.

[11] S. Cakaj, "Modulation Index Application for Satellite Adjacent Downlink Interference Identification", The 6th European Conference on Antennas and Propagation EUCAP 2012, IEEE, March 26-30, 2012 Prague, Czech Republic, pp. 2000-2004.

[12] S. Cakaj, W. Keim, K. Malaric, "Intermodulation by Uplink Signal at Low Earth Orbiting Satellite Ground Station", $18^{\text {th }}$ International Conference on Applied Electromagnetics and Communications, ICECom, IEEE, 12-14 October 2005, Dubrovnik, Croatia, pp. 193 - 196.

[13] S. Cakaj, K. Malaric, A. L. Schotlz, "Modelling of Interference Caused by Uplink Signal for Low Earth Orbiting Satellite Ground Stations", $17^{\text {th }}$ IASTED International Conference on Applied Simulation and Modelling, ASM 2008, June, 23 -25, 2008, Corfu, Greece, pp. 187-191.

[14] G.D. Gordon, W.L. Morgan, 'Principles of communication satellites", John Wiley \& sons, Inc. 1993.

[15] H. Curtis, "Orbital Mechanics for Engineering Students", Elsevier aerospace engineering series, pg. 55, 1998.

\section{AUTHOR PROFILE}

Shkelzen Cakaj has received his BSc and MSc

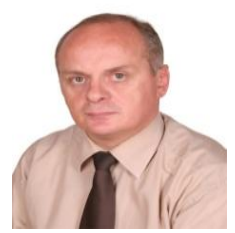
degrees from Prishtina University in Kosovo. Since 2003 is cooperating with Institute for Communication and Radio - Frequency Engineering at the Technical University in Vienna, where he has prepared his Master Thesis related to the performance of the ground satellite station in July, 2004. He was awarded a PhD in area of satellite communication from Zagreb University in January 2008 with whom he has continued technical associations. He has attended courses on satellite communication and spectrum management at USTTI. He was awarded as Fulbright scholar researcher in 2009 at NOAA (National Oceanic and Atmospheric Administration) at Maryland, USA. He is the author of 45 papers published in worldwide conferences and journals; mostly IEEE. His area of interest is the performance of satellite ground stations for scientific satellites. He is working at Post and Telecommunication of Kosovo and lecturing satellite communications for master students at Prishtina University, Kosovo and Polytechnic University of Tirana, Albania. 